\title{
Spatial attention in Ponzo-like patterns
}

\author{
ALEXANDER W. PRESSEY and DELMAR EPP \\ University of Manitoba, Winnipeg, Manitoba, Canada
}

\begin{abstract}
A variant of Ponzo's pattern was devised by drawing converging oblique lines at the ends of each of two horizontal lines that were located directly one above the other. Pairs of converging or diverging fins (either between or outside the horizontal shafts) were systematically removed. The results showed that, as predicted by integrative field theory, oblique lines between the shafts produced more distortion than did oblique lines outside the shafts. Also, it was shown that the attentive field construct in integrative field theory was crucial for predicting patterns of means and variances in this experiment. The modified Ponzo effect was shown to have much in common with the classical Müller-Lyer illusion. The subtle differences between the two were explained by proposing that the size of the optimum attentive field was larger in the Ponzo tasks than in the Müller-Lyer tasks because expansion fins are found on both shafts in the Ponzo-like figure.
\end{abstract}

The configuration shown in Figure $1 \mathrm{~A}$ is named after Mario Ponzo, who described the effect in the early part of this century (Ponzo, 1912). The horizontal line near the apex of the long oblique lines appears larger than the physically equal horizontal line beneath it. The explanation of the Ponzo effect that has dominated textbook literature (e.g., Sekuler \& Blake, 1990) is attributed to Richard Gregory (1963), although a similar position was developed by Tausch in 1954. Gregory's view is called an "inappropriate constancy scaling" theory because it proposes that three-dimensional visual information is filtered through a cognitive strategy in which near objects are perceptually reduced and distant objects are perceptually enlarged. This Kant-like category distorts information in order to extract the object character from the proximal stimulation.

There are many reasons for the popularity of Gregory's (1963) explanation, at least two of which deserve to be elaborated. First, it appeals to a reemerging functionalism, which characterizes modern cognitive psychology because of the ease with which analogues can be found in nature. Many descriptions of Ponzo's pattern are accompanied by natural scenes such as railroad tracks that recede into the distance. It is then argued that the oblique lines act as cues to depth which induce the perceptual system to treat the two-dimensional representation inappropriately as a three-dimensional view. Therefore, the upper horizontal line appears farther away from the observer than the lower one, with the result that size-

This research was supported by a grant from the University of Manitoba Research Committee of Senate. We are indebted to Chris Pressey, who wrote the program for displaying stimuli and who modified Bernd Kersten's Müller-Lyer prediction program to apply to the modified Ponzo task used in this study. Correspondence should be addressed to A. W. Pressey, St. John's College, The University of Manitoba, Winnipeg, Manitoba, Canada R3T 2M5 (e-mail: pressey@uofmcc).

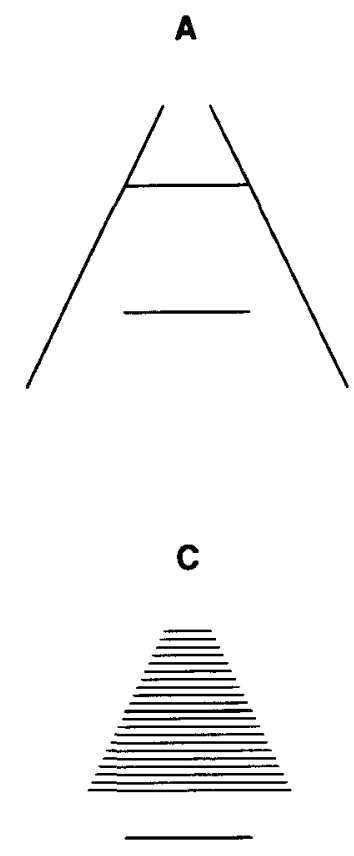

$\mathbf{B}$

Figure 1. (A) Ponzo's configuration. (B) A variant of Ponzo's configuration. (C) A parallel-lines version of Ponzo's configuration. (D) A minimum attentive field superimposed on a variant of Ponzo's pattern.

constancy mechanisms enlarge the upper (farther) shaft and shrink the lower (nearer) one.

A second reason for the strong impact of Gregory's (1963) theory is that it removes the so-called "optical-geometric illusions" from the realm of perceptual error and locates them in the realm of ordinary perception. Many researchers who study illusions defend their programs by arguing that such illusions might enlighten us about normal perception (e.g., Jordan \& English, 1989). Such a proposition is rooted in a metaphysics of 
naive realism and a copy theory of perception which proposes that our percepts are faithful representations of reality. According to this view, illusions are errors; they are abnormal and most likely due to some anomaly in the physics of light or in the anatomy, physiology, and engineering of the sensory-brain system. Gregory's proposal, on the other hand, invites us to understand illusions to be the product of a properly functioning system that, under esoteric or unnatural conditions, operates inappropriately. Thus, an illusion is not a flawed perception but an innocent deception.

Gregory's (1963) point of view represents a fundamental shift in our approach to perception, but the specific theory suffers from what seems to be an insurmountable problem. It is difficult to generate unambiguous predictions, and those that are generated often are not verified (Jordan \& Randall, 1987; Reardon \& Parks, 1983; Waite \& Massaro, 1970). One fact that is especially difficult to rationalize is that reversals in direction of distortion can occur. In Ponzo's figure, reversals can be induced either by making the angle between the oblique lines very large (Pressey, Butchard, \& Scrivner, 1971) or, for some observers, by shifting the bottom shaft to a position above the apex (Pressey, 1974b). An example of the kind of difficulty that Gregory faces is provided in Figure 1B, in which Ponzo-like distortions are present but in which depth cues are ambiguous. Gregory would have to argue that in this case the upper shaft is treated as if it were nearer to the observer than the lower shaft, but it is neither explicitly or intuitively obvious why this should be so.

\section{Integrative Field Theory: \\ The Descriptive Version}

An alternative to Gregory's (1963) explanation (initially called assimilation theory, but now called integrative field theory [IFT]; Pressey \& Smith Martin, 1990) proposes that Ponzo's illusion is a compound of a shrinkage and an expansion form of a Müller-Lyer figure, which in turn is a complicated version of a parallel-lines illusion, as shown in Figure 1C (Pressey, 1971; Pressey \& Murray, 1976). The upper horizontal shaft is integrated with implicit horizontal lengths that are defined by the oblique lines. In this case, integration takes the form of averaging, in which the focal stimulus regresses to, and becomes more like, the context. The notion of averaging is one of the most widely used ideas in psychology, and it has been called a central tendency effect, regression to the mean, assimilation, leveling, pooling, lumping, and entropy. Moreover, those who argue that illusions of size are caused by a framing effect (Brigell, Uhlarik, \& Goldhorn, 1977; Künnapas, 1955) would endorse the idea that the length of a line "becomes like" the length of the frame.

However, it is clear that averaging alone cannot explain the Ponzo effect shown in Figure 1C because, in this case, the average of the series is equal to the length of the focal line. The resolution of this difficulty lies in understanding that percepts are not pictures that provide homogeneous weightings to all portions of the two- (or three-) dimensional visual array. The observer attends to certain parts of the display to a greater degree than to others, with the result that certain kinds of information will be more heavily weighted than others in the formation of a percept. Furthermore, it is contended that an a priori estimate of such spatial deployment of attention can be made by assuming that the observer is a rational operator whose intention it is to conform to the objectives of the task as set by the instructions. Thus, in the Ponzo task, the objective is to compare the upper and lower horizontal lines. To carry out the task at all, it is necessary to assume that the four end points of the two shafts must be processed. An inscription of these points by a circle defines a minimum attentive field ${ }^{1}$ whose midpoint is the center of the circle. Figure 1D illustrates this notion and shows that the expansion fins of the Ponzo pattern fall within this minimum field and are closest to the center of attention. There is, of course, a potential for the shrinkage fins outside the minimum field to affect the focal shaft but, because they are farther from the center of attention, the probability of processing them is less than the probability of processing the expansion fins, with the result that the net effect is one of expansion.

It is not an easy matter to test the validity of the notion of an attentive field. We have adopted two approaches. The first is a qualitative-rational one in which an attempt is made to show how such diverse effects as Ponzo's distortion of length, Poggendorff's distortion of direction, and Wundt's illusion of area are explained by an attentive field (Pressey, 1971); how reversals in direction of distortion can be induced (Pressey, 1971, 1974b, Pressey et al., 1971); and how new illusory targets can be generated (Pressey, 1971, 1974a).

An example of this approach is provided by the "pop cup" illusion ${ }^{2}$ shown in Figure 2. When two identical (trapezoidal) cups are placed one above the other, the upper cup appears smaller than the lower one. It is proposed that the calculation of area takes into account the apparent size of the base and the rim of each cup. According to IFT, the center of the attentive field is located between the base of the upper cup and the rim of the lower one. In the calculation of the area of the upper cup, therefore, the (short) base will be excessively weighted as compared with the upper rim and the computed area will be underestimated. Conversely, the (large) rim of the lower cup will be heavily weighted as compared with the base, with the result that the area will be overestimated. Thus, in the global comparison between the two cups, the lower cup will appear larger than the upper one.

The second approach has involved the development of a mathematical model with precise, operationally defined descriptions of the hypothetical constructs. This model has been applied to specific test situations. Exact predictions were generated, empirical functions were established, and the fit between obtained and predicted functions was assessed. This tactic has been applied to Ponzo (Pressey et al., 1971), Müller-Lyer (Pressey, 


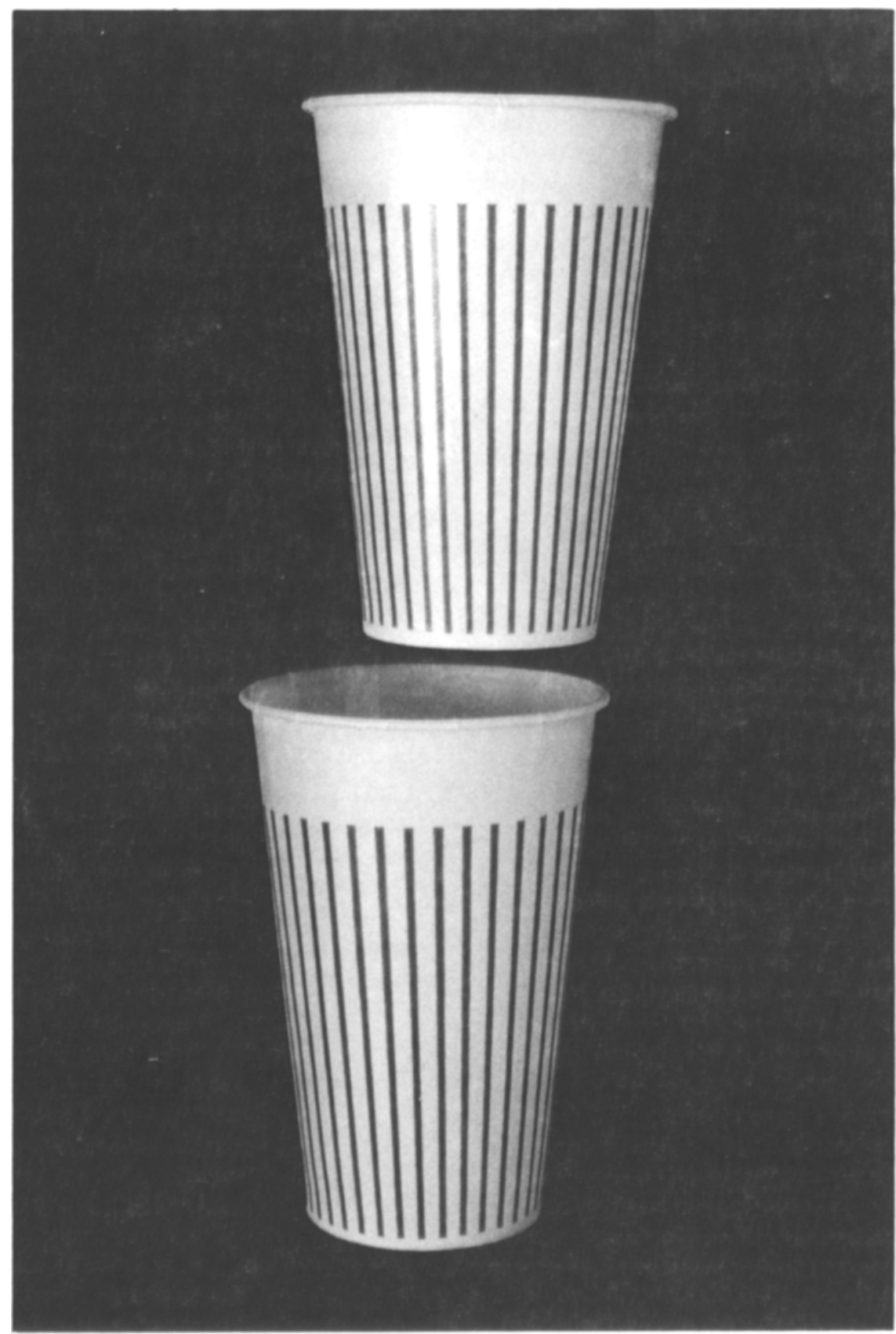

Figure 2. The "pop cup" illusion. The upper cup appears smaller than the cup beneath it.

Di Lollo, \& Tait, 1977), and Baldwin (Pressey \& Wilson, 1980) configurations. The qualitative and quantitative evidence appears to confirm the idea that spatial deployment of attention is essential in explaining diverse perceptual effects, and several groups of investigators have recognized the utility of the idea (Jaeger, 1985; Jordan \& English, 1989; Jordan \& Randall, 1987; McLellan, Bernstein, \& Garbin, 1984; Restle \& Decker, 1977).

A potentially powerful strategy for assessing the role of attention deployment is to measure the effects of amputating selected features of a display. Often such a tactic has been employed in research on Poggendorff's illusion (e.g., Weintraub \& Krantz, 1971), but it has been exploited only rarely in other configurations (Pressey, 1974b; Warren \& Bashford, 1977). A recent experiment by Larsen and Garn (1988) demonstrates the utility of selective amputation. They employed a compound version of Müller-Lyer's illusion in which the two focal shafts were horizontally oriented and located one directly above the other. Shrinkage and expansion fins were removed, either from between or from outside the shafts. The results indicated that removing the fins from between the 
shafts had a greater effect than did removing them from outside the shafts and that the shrinkage illusion was more strongly affected by this differential amputation.

It is possible to selectively remove fins from between or from outside the two focal shafts in Ponzo-like figures, just as Larsen and Garn (1988) did within the Müller-Lyer pattern. An application of integrative field theory to such amputated versions reveals several interesting predictions, the most noteworthy of which is that the full Ponzo pattern will not yield the largest distortion. Specifically, removing fins from outside the shafts (see Figure 4, C+D and $\mathrm{G}+\mathrm{H}$ ) should enhance the effect as compared with the full figure (Figure 4, A+B). Such an outcome would not be anticipated by a perspective or an inappropriate constancy scaling theory of Ponzo's effect. The main purpose of this study was to determine whether the Ponzo illusion could be enhanced by removing oblique lines that are external to the focal shafts.

A second aim was to assess how well the quantitative version of IFT predicts the effects of selective amputation. A variable of fin length was added to complicate both predicted and obtained functions.

\section{Integrative Field Theory:}

\section{The Quantitative Version}

According to IFT, judged size $\left(J_{s}\right)$ is a weighted average of the size of the standard stimulus $(S)$ and the size of the contextual line(s) (C). This idea is expressed by the following equation:

$$
J_{s}=P_{c} \cdot C+P_{s} \cdot S,
$$

where $P_{c}$ is the probability that $C$ is sampled, $P_{s}$ is the probability that $S$ is sampled, and $P_{s}=\left(1-P_{c}\right)$.

In the parallel-lines target, if $J_{s}$ is the arithmetic average of $C$ and $S$, this would be expressed by

$$
J_{s}=.5 \cdot \mathrm{C}+.5 \cdot \mathrm{S} .
$$

It was found that a correlate of Equation 2 is a strong predictor of empirical functions relating the Müller-Lyer and Poggendorff illusions to several stimulus variables (Pressey, 1972). However, it was also found that better predictions could be made if both the distance between $C$ and $S$ and the position within the attentive field were included (Pressey et al., 1971; Pressey \& Murray, 1976).

The contribution of attention deployment is calculated by measuring the distance from the center of attention to the tip of a contextual magnitude $\left(D_{c}\right)$ as shown in Figure 3 and then relating this distance to the radius $(r)$ of a hypothetical circular field. The center of this field is operationally defined as the midpoint between the most distal elements of the standard and comparison lines as set by the instructions for the task. Thus, the probability that $C$ contributes to the judgment of the standard line is given by

$$
1-\frac{D_{c}}{r}
$$

In performing computer simulations of actual experiments, several values of $r$ are sampled. This is because

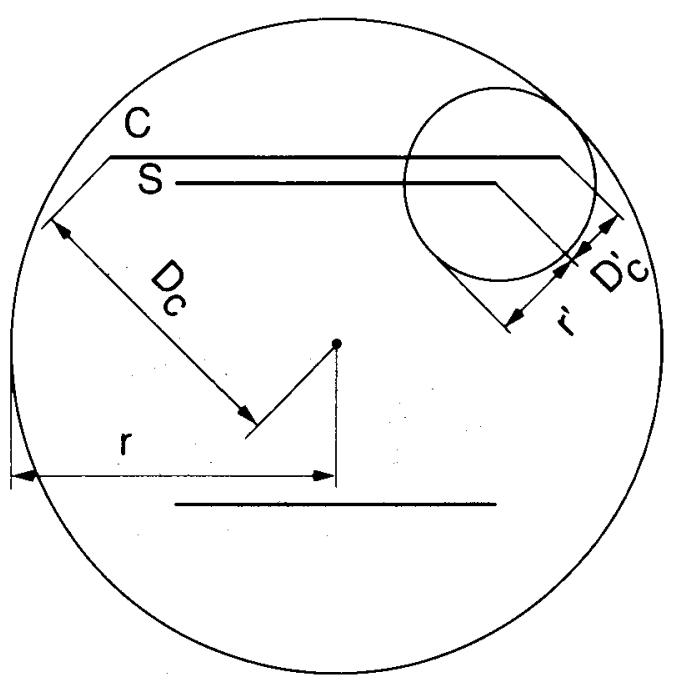

Figure 3. The parallel-lines display upon which the quantitative version of integrative field theory is based. $S$ is the standard line (which, in most cases, is $50 \mathrm{~mm}$ long), and $C$ is the contextual line. The comparison line is located $100 \mathrm{~mm}$ below the standard line and, on average, appears directly below it. The large circle represents an attentive field. The center of this field is depicted by the small dot, which is the midpoint between the most extreme points of the standard and comparison lines. $D_{c}$ is the distance from the center of the attentive field to the most distal point of the contextual line, and $r$ is the distance from the center of the attentive field to the edge of that field. In most simulations, $r$ varied from 66 to $106 \mathrm{~mm}$ in steps of $10 \mathrm{~mm}$. The small circle represents an interactive field. The tip of the standard line is the center of this field, and in most simulations, radii ranged from 10 to $40 \mathrm{~mm}$ in steps of $10 \mathrm{~mm}$. $D_{c}^{\prime}$ is the distance from the center of the interactive field to the most distal point of the contextual line, and $r^{\prime}$ is the radius of a hypothetical interactive field.

$r$ is presumed to be an organismic variable that reflects a particular style of deploying attention across the stimulus field. For present purposes, three sizes of field can be defined: (1) minimum field, which is the smallest radius that encompasses all and both of the standard and comparison lines; (2) optimum field, which is the radius that provides the best fit to empirical data; and (3) maximum field, which is the size that must be postulated to calculate the optimum field. In most simulations, five radii of attentive field (ranging from 66 to $106 \mathrm{~mm}$ in steps of $10 \mathrm{~mm}$ ) are sampled. These sizes are directly related to the parameters of the experimental targets as defined in the legend of Figure 3. However, where $S$, or the distance between $S$ and the comparison line, varies, then the values of $r$ are multiplied by a ratio of the new minimum field to the original minimum field (Pressey \& Di Lollo, 1978).

The attentive field is insufficient to account for several nuances in empirical trends, and it is necessary to postulate that distance between $C$ and $S$ per se plays a role in effecting distortion (Pressey \& Murray, 1976). A second field, called the interactive field, is proposed. For various reasons, discussed by Pressey and Murray, the center of this field is located at a tip of the standard line, as shown 
in Figure 3. The interactive field also specifies the probability that $C$ is sampled during the judgment of $S$, and it is derived by the equation

$$
1-\frac{D_{c}^{\prime}}{r^{\prime}}
$$

where $D^{\prime}{ }_{c}$ is the distance from the tip of $S$ to the tip of $C$ and $r^{\prime}$ is the radius of the hypothetical interactive field.

Like the attentive field, it is assumed that the interactive field reflects individual differences in perceptual stylethis time in spatial discriminability - so that individuals with small fields are considered to be highly discriminative, whereas those with large fields are considered to be poor discriminators. In simulations using the standard target described in Figure 3, four sizes of field with radii varying from 10 to $40 \mathrm{~mm}$ in steps of $10 \mathrm{~mm}$ are sampled.

When contributions of both the attentive and interactive fields are considered simultaneously, the new value of $P_{c}$ in Equation 1 becomes

$$
P_{c}=\left(1-\frac{D_{c}}{r}\right)\left(1-\frac{D_{c}^{\prime}}{r^{\prime}}\right)
$$

However, Equation 5 applies only to the case in which there is one contextual line, as in the parallel-lines illusion. Where complicated patterns such as Ponzo's figure are used, these patterns are converted to a series of parallel lines, as shown in Figure 1C. A new value of $J_{s}$ is calculated which represents a weighted average of all the contextual lines so sampled. The new equation is given by

$$
\begin{aligned}
J_{s}= & \frac{1}{N} \sum_{j=1}^{N}\left(1-\frac{D_{c j}}{r}\right)\left(1-\frac{D_{c j}^{\prime}}{r^{\prime}}\right) \cdot C_{j} \\
& +1-\left[\left(1-\frac{D_{c j}}{r}\right)\left(1-\frac{D_{c j}^{\prime}}{r^{\prime}}\right)\right] \cdot S,
\end{aligned}
$$

where $D_{c j}$ is the distance from the center of the attentive field to the most distant point of contextual magnitude, $j ; r$ is the radius of the attentive field; $D_{c j}^{\prime}$ is the distance from the tip of the standard line to the tip of contextual magnitude, $j ; r^{\prime}$ is the radius of the interactive field; and $N$ is the total number of contextual magnitudes sampled by this estimation procedure, all of which fall entirely within both the attentive and interactive fields. ${ }^{3}$

The double Ponzo figures used in this experiment are shown in Figure 4. The amputations conform to those used by Larsen and Garn (1988) in the Müller-Lyer figure. Patterns $A+B$ are the full figures; in $C+D$, an external shrinkage fin is removed; in $E+F$, an internal shrinkage fin is removed; in $\mathrm{G}+\mathrm{H}$, an external expansion fin is removed; and in $\mathbf{I}+\mathbf{J}$, an internal expansion fin is removed.

A computer program used by Pressey and Kersten (1989) was modified to calculate predictions for a Ponzo pattern. The angle between the fin and the shaft for the shrinkage form was $52.5^{\circ}$, and for the expansion fin it was $127.5^{\circ}$. The shafts were $46 \mathrm{~mm}$ long, and the fins varied from 5 to $25 \mathrm{~mm}$ in steps of $5 \mathrm{~mm}$. The radii of

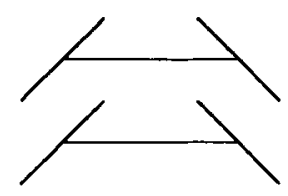

$A+B$

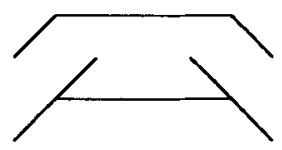

$C+D$
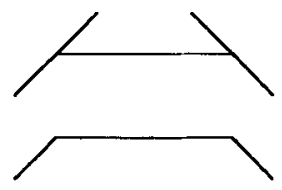

$E+F$
$\mathbf{G}+\mathbf{H}$

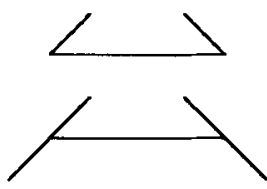

$\mathbf{I}+\mathbf{J}$

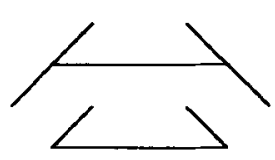

Figure 4. Stimuli used in the current study. The second letter in each pair refers to the case in which positions of the upper and lower targets are reversed.

the hypothetical attentive fields used for prediction ranged from 34 to $74 \mathrm{~mm}$ in steps of $10 \mathrm{~mm}$, and for the hypothetical interactive fields they ranged from 10 to $40 \mathrm{~mm}$ in steps of $10 \mathrm{~mm}$.

The predicted pattern of results is shown in Figure 5. Two points are of interest. First, it is predicted that two

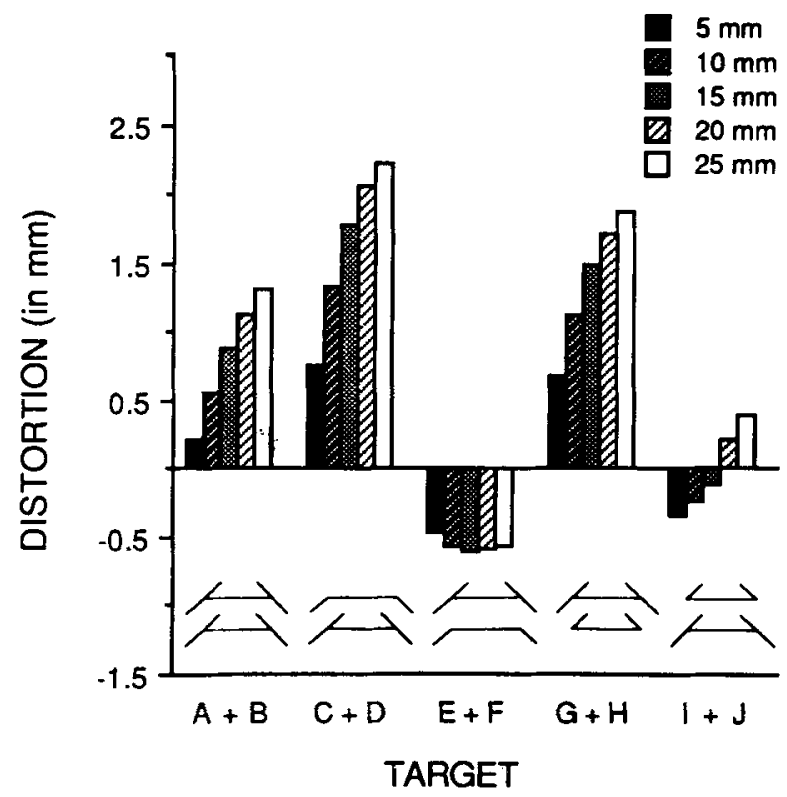

Figure 5. Predicted distortion in modified Ponzo pattern as a joint function of type of fin deleted and length of fin. 
amputated variants $(C+D$ and $G+H)$ will produce larger effects than will the full form. This is unlike the MüllerLyer effect, which shows a decrease whenever any fin is amputated (Larsen \& Garn, 1988; Pressey \& Pressey, 1992). Second, a small reversed effect should occur when the fins between shafts are removed and, in the case of inner shrinkage fin removal, increasing fin length should increase somewhat the degree of reversal.

\section{METHOD}

\section{Subjects}

Twenty-two women and 9 men from an introductory psychology course at the University of Manitoba participated to fulfill a course requirement. Only those with good vision, with or without corrective lenses, were asked to volunteer.

\section{Apparatus}

Figures were displayed on a black-and-white Electrohome monitor in which 100 pixels yielded a $46-\mathrm{mm}$ line. Five sets of 10 targets, similar to those shown in Figure 4, were used in which the two horizontal shafts were $46 \mathrm{~mm}$ long. The perpendicular distance between shafts was $50 \mathrm{~mm}$, the angle between the shrinkage fin and the horizontal shaft was $52.5^{\circ}$, and the angle between the expansion fin and the shaft was $127.5^{\circ}$. The five sets of targets differed in the length of fins, which ranged from 5 to $25 \mathrm{~mm}$ in steps of $5 \mathrm{~mm}$.

The length of the lower horizontal shaft could be increased or decreased symmetrically in 2-pixel steps. Right and left buttons on an external handset controlled increments and decrements, and a third (center) button advanced the display.

A computer controlled the randomization and display of targets, response acquisition, and scoring. Ambient illumination in the testing room was maintained at approximately $150 \mathrm{~lx}$, and the ratio of brightness between figures and screen background was approximately $20: 1\left(90.7\right.$ to $\left.4.5 \mathrm{~cd} / \mathrm{m}^{2}\right)$. The observers used an adjustable chinrest to view the screen from a distance of approximately $45 \mathrm{~cm}$.

\section{Procedure}

The observers were seated in front of the monitor, and a target from the appropriate set was randomly selected and displayed. The two horizontal shafts were pointed out, and the experimenter stated that they were physically equal but appeared of different length because of the oblique lines at the ends of the shafts. The observers were asked to press repeatedly an appropriate button until the two horizontal shafts appeared to be equal in size. When they were satisfied with their judgment, they were told that the center button was to be depressed to present a new display. It was emphasized that the observers were to equate only the horizontal size of the two shafts. Specifically, they were asked to refrain from lining up the endpoints of the shafts.

The order of presenting the five sets of targets was randomized, and each subject performed under all conditions. The subjects proceeded in a self-paced manner, and the task was completed in about $15 \mathrm{~min}$.

\section{RESULTS AND DISCUSSION}

In each set, the raw score from target $B$ was subtracted from target A; D from C; $F$ from $E ; G$ from $H$; and $J$ from 1 . The resulting values were divided by 2 to obtain a mean score for each pair. These scores were subjected to a $5 \times 5$ within-subject analysis of variance (ANOVA) in which there were five types of Ponzo-like targets and five lengths of oblique lines. The type of target $[F(4,120)$
$=114.3, p<.05]$, length of oblique lines $[F(4,120)=$ $31.9, p<.05]$, and the interaction effect $[F(16,480)=$ $11.8, p<.05]$ were all statistically significant.

The results, illustrated in Figure 6, indicate a strong similarity to the predicted patterns shown in Figure 5. In comparing means of distortion across subjects between predicted and observed scores, the Pearson $r$ between the two sets of data points was $.97, d f=23, p<.05$. However, it should be noted that a reversal in the direction of distortion predicted for targets $\mathrm{E}+\mathrm{F}$ and $\mathrm{I}+\mathrm{J}$ in Figure 5 was not reliably found in the obtained data. We have no explanation for the misprediction at this time.

In the first simulation employing IFT (Pressey et al., 1971), it was discovered that the quantitative model correctly predicted inverted-U functions relating a Ponzo illusion to angle of oblique lines for both means and variances. In other words, means and variances were correlated in both the predicted and obtained data. To confirm these findings, the standard deviations of the data from each matrix of 20 theoretical scores (corresponding to contributions of five attentive and four interactive field sizes) were calculated. The predicted scores are shown in Figure 7. A comparison of the predicted variability of scores with the predicted means (shown in Figure 5) indicates that there is a similarity between the two patterns $[r=$ $.77, d f=23, p<.05$ ], and this verifies that IFT correctly predicts correlated functions.

The standard deviations of the obtained data are shown in Figure 8. Clearly, the predicted patterns of variability are similar to the obtained patterns $(r=.90, d f=23$, $p<.05$ ), and they are similar to the obtained patterns of means $(r=.80, d f=23, p<.05)$.

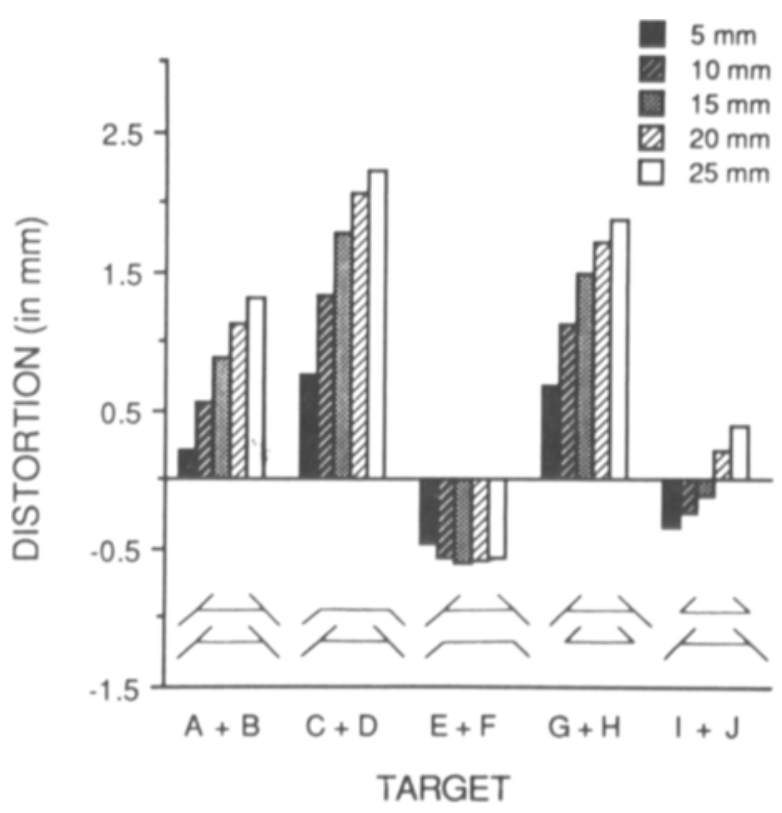

Figure 6. Obtained distortion in modified Ponzo pattern as a joint function of type of fin deleted and length of fin. 


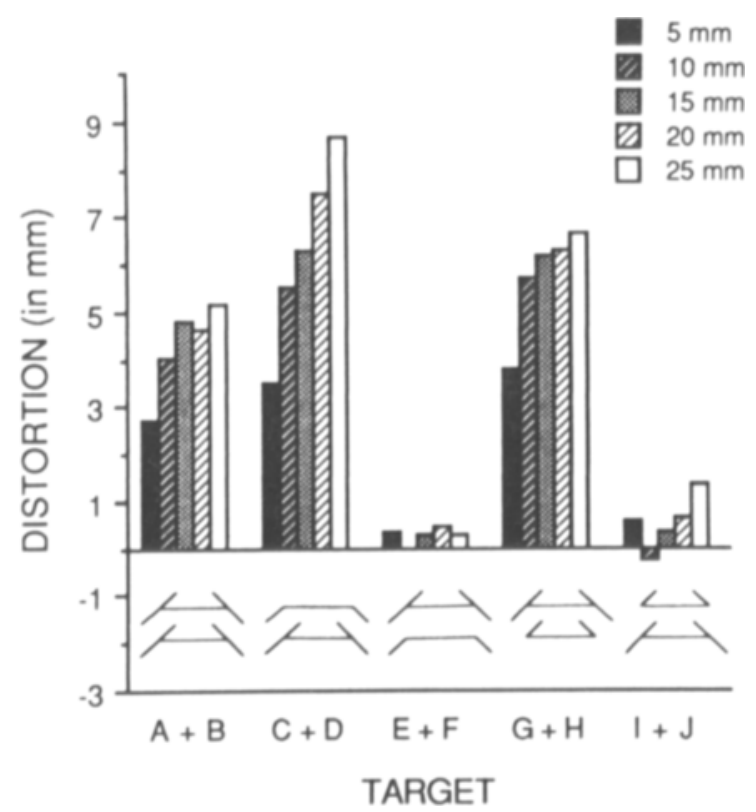

Figure 7. Predicted standard deviation in modified Ponzo pattern as a joint function of type of fin deleted and length of fin.

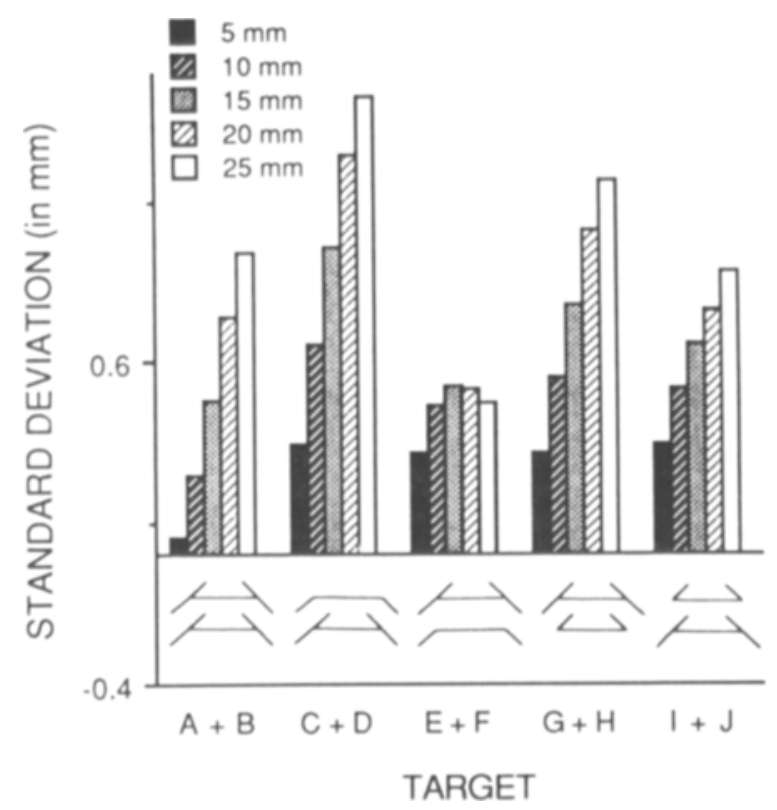

Figure 8. Obtained standard deviation in modified Ponzo pattern as a joint function of type of fin deleted and length of fin.

\section{Comparing Ponzo and Müller-Lyer Figures}

The complete Ponzo pattern used in this study is juxtaposed in Figure 9 with a Müller-Lyer pattern used in previous studies (Larsen \& Garn, 1988; Pressey \& Pressey, 1992). It is clear that the Ponzo-like figure is produced by exchanging the outer contralateral fins of the MüllerLyer configuration. In other words, if the four pairs of fins (shrinkage outside shafts, shrinkage between shafts, expansion outside shafts, and expansion between shafts) are considered to be elements of the display, then the Müller-Lyer and Ponzo patterns have common elements but different configurations. Furthermore, if configuration plays no role, then it should be possible to exactly predict Müller-Lyer data from Ponzo data, and vice versa.

An experiment using Müller-Lyer figures in which pairs of fins were amputated in a manner identical to the one used in the present study has been conducted in our laboratory (Pressey \& Pressey, 1992). Eighteen subjects were tested under conditions identical to the present experiment except that Müller-Lyer figures with 30-mm fins were also used. For present purposes, the data from the $30-\mathrm{mm}$ fins were omitted. The raw data from both experiments were recalculated as deviations from complete figure (i.e., from Figures $A+B$ ) in the expected direction. Thus, for example, removing the outer shrinkage fins in Müller-Lyer Figures $C+D$ should result in a reduction of illusion, and in such a case a positive score would result. On the other hand, for the equivalent Ponzo figure, removal of the outer shrinkage fins should enhance the illusion, and so a positive score also would be assigned. In this way the contribution of each pair of fins could be ascertained.

In the first analysis, the contributions of each of the components of the Ponzo illusion were determined and were added in such a way as to simulate full and amputated versions of Müller-Lyer patterns. The predicted Müller-Lyer scores are shown in Figure 10, in which obtained data are also illustrated. The two patterns are very similar $[r=.95, d f=3, p<.05]$ but the levels are different. In a second analysis, components derived from Müller-Lyer targets were added in such a fashion as to simulate the Ponzo figures used in this experiment. The results are shown in Figure 11. Again, the predicted and obtained patterns are very similar $[r=.99, d f=3$, $p<.05]$ but the levels differ. Thus, there does not appear to be an equivalence between identical components when they are organized as Müller-Lyer or as Ponzo configurations.

\section{A}

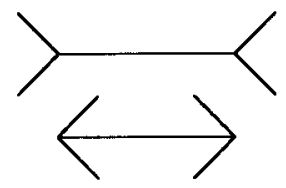

B

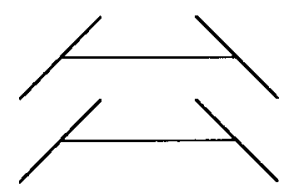

Figure 9. A comparison of a composite Müller-Lyer figure (A) with a variant of Ponzo's pattern (B). 


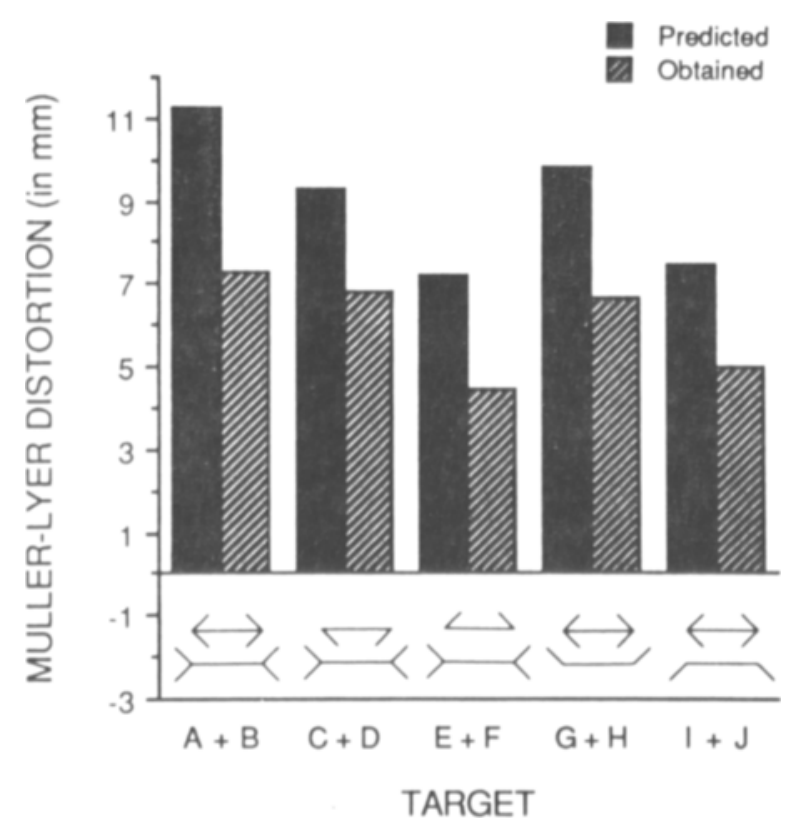

Figure 10. Predicted distortions in Müller-Lyer targets based on data obtained with Ponzo patterns. The obtained Müller-Lyer data are from Pressey and Pressey (1992).

To further explore the role of stimulus configuration, the manner in which each of the four components in both Müller-Lyer and Ponzo figures was affected by increases in fin length was analyzed. As illustrated in Figure 12, in every case the components in the Ponzo figure showed greater deviation from the full figure than did the analogous components in the Müller-Lyer configuration $[F \mathrm{~s}(1,47)>5.45, p<.05]$. Also, in every case but one, the effect of fin length was significant $[F \mathrm{~s}(4,188)>3.11$, $p<.05]$ but the interaction was not. The one exception was the shrinkage fin outside the shafts, which exhibited a nonsignificant effect due to fin length but a large interaction effect $[F(4,188)=8.3, p<.05]$. The trends shown in Figure $12(C+D)$ indicate that increasing fin length in the Müller-Lyer figure did not add to the total distortion, whereas in the Ponzo figure it did.

The interaction shown in Figure $12(C+D)$ can be explained in terms of the attentive field as proposed by IFT. Simulation techniques applied to Müller-Lyer figures have led us to conclude that increasing the length of expansion fins widens the radius of the field, but increasing fin length for the shrinkage fin has no effect on the optimum size of the field (Pressey \& Pressey, 1992). In other words, the attentive field for shrinkage fins "hugged" the ends of the shaft, whereas the field for the expansion form was captured by the tips of the fins. Thus, it seems that attentive field size can be altered by contextual features, and it is possible that, in the Ponzo configuration, the field increases with increases in the length of both shrinkage and expansion fins. But now the question is why the field for the shrinkage fins is tied to the shaft in the MüllerLyer figure but to the fins in the Ponzo figure. One pos- sible solution arises from a close examination of Ponzo's pattern (see Figure 9). Here, the shrinkage fins are coupled with expansion fins, and physical increases in the shrinkage fins are coupled with physical increases in the expansion fins. So if, as we already know, increasing expansion fins increases attentive field size and if the field remains roughly circular, then the proximal shrinkage fins will also fall in increasingly larger attentive fields. This means that they will contribute increasingly larger illusions as fin length increases. In other words, shrinkage fins do not directly alter the size of the field in either the Müller-Lyer or Ponzo configurations, but in the Ponzo pattern, field size for the shrinkage fin is enlarged indirectly by the adjacent expansion fin.

This explanation for the nonequivalence of the external shrinkage fins in Ponzo and Müller-Lyer configurations can be tested by the quantitative version of IFT. In a recent study (Pressey \& Pressey, 1992), the program for predicting distortion was altered in two ways. First, predictions for five Müller-Lyer targets were generated under all possible combinations of attentive field size assigned separately to shrinkage and expansion forms. Then a correlation subroutine compared these hypothetical functions with an empirically obtained function, and the combination yielding the highest coefficient was selected. (It was this procedure that showed that attentive field size is determined by the configurational factors.) A similar modification was made in the Ponzo program used in this experiment, and optimum sizes of fields were established separately for shrinkage and expansion forms. Six sizes of attentive field were used in a series of successive approximations until a unique best-fit solution was

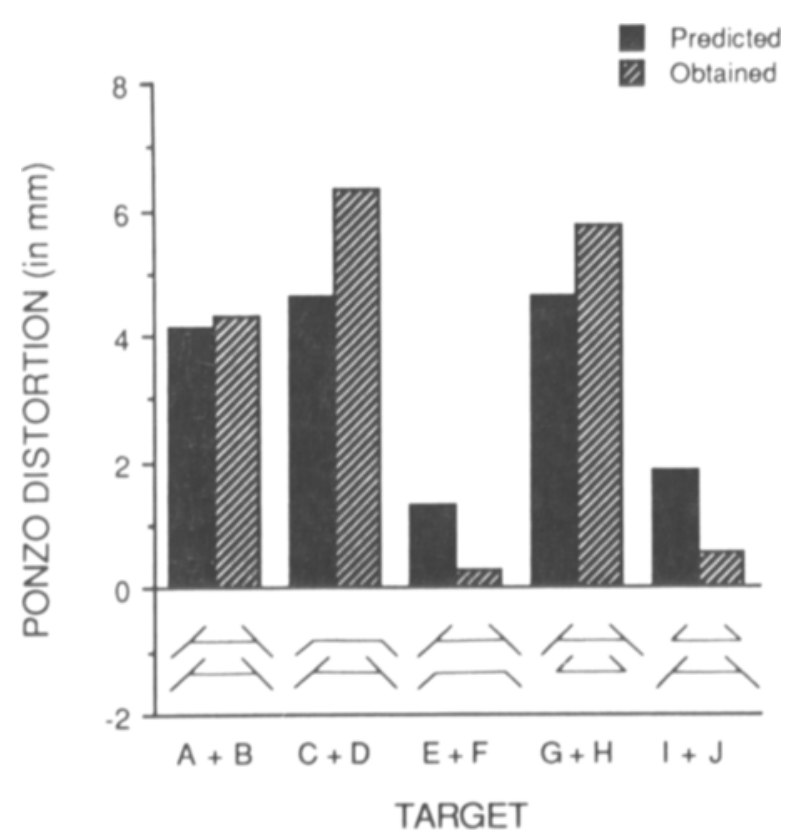

Figure 11. Predicted distortions in Ponzo-like targets based on data obtained with Müller-Lyer patterns. The obtained Müller-Lyer data are from Pressey and Pressey (1992). 

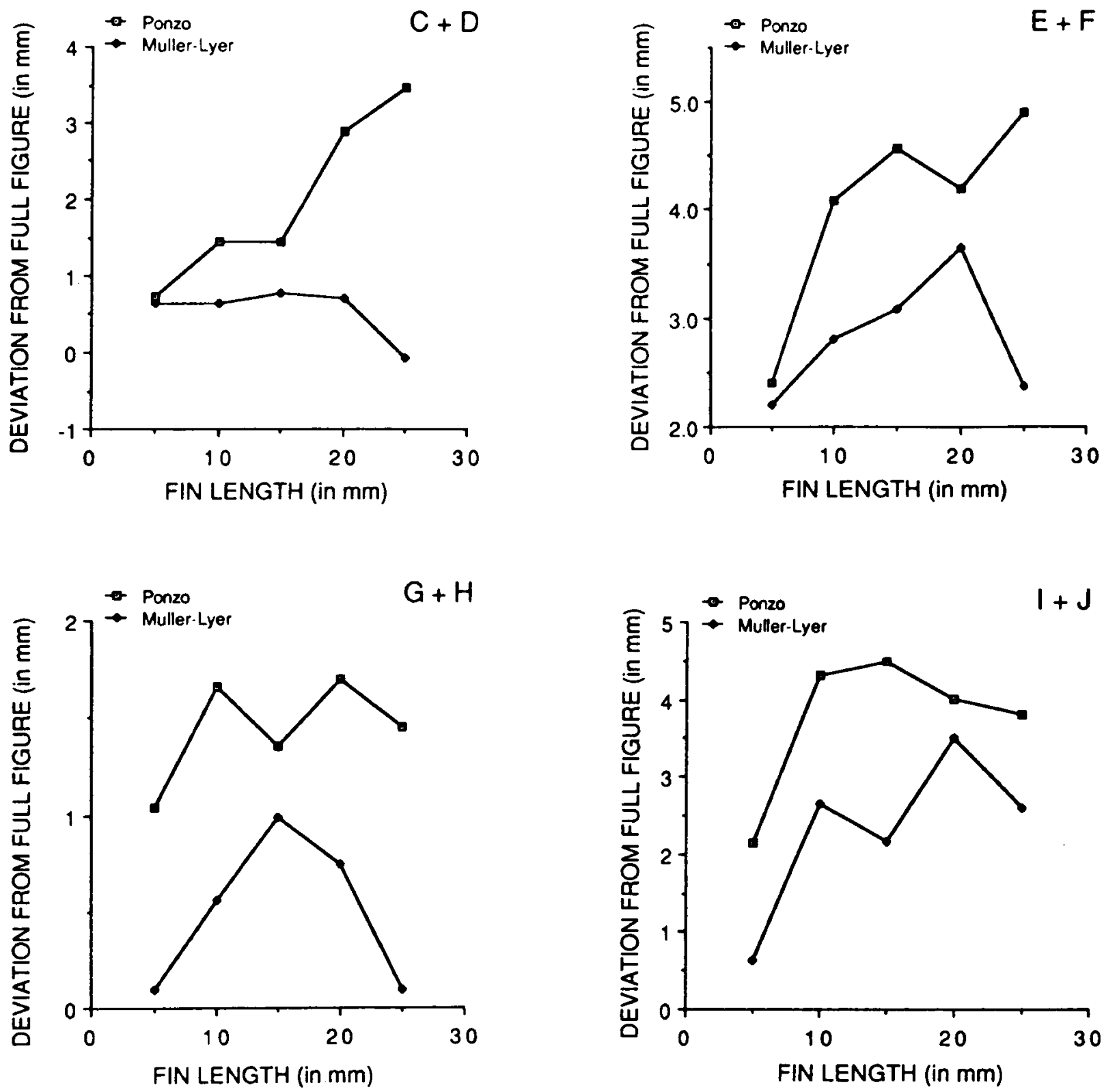

Figure 12. Effect of fin length and type of fin deleted on reducing distortion in Ponzo and Müller-Lyer configurations. In $C+D$, the external shrinkage fin is removed; in $\mathbf{E}+\mathbf{F}$, the internal shrinkage fin is removed; in $\mathbf{G}+\mathbf{H}$, the external expansion fin is deleted; and in $\mathbf{I}+\mathrm{J}$, the internal expansion fin is deleted.

attained. However, while this procedure had worked well for Müller-Lyer figures (i.e., a single combination yielded the highest coefficient), for Ponzo figures, identical coefficients were found for a cluster of combinations. On occasion, this cluster extended to include very large values of hypothetical field size. Therefore, it was decided to select the smallest value of field size that provided the best fit to the data. The results are shown in Figure 13.

Inferred field sizes are similar to those found for MüllerLyer figures in two respects: (1) in both configurations, field size for the expansion form generally is larger than for the shrinkage form, and (2) field size for the expansion fins increases with fin length in both Müller-Lyer and Ponzo patterns. The major difference is that the inferred field size for the external shrinkage fins in the Ponzo illusion increases with increases in fin length, but remains constant in Müller-Lyer figures. Thus, the solution for the configurational difference proposed earlier, namely, that it is due to differences in attention, is reinforced by the inferential techniques based on the quantitative version of IFT.

The fact that component parts behave differently when they are organized as wholes called Ponzo figures than when they are organized as wholes called Müller-Lyer figures is, of course, the classic problem of gestalts. One interpretation of the claim that the whole is different from 


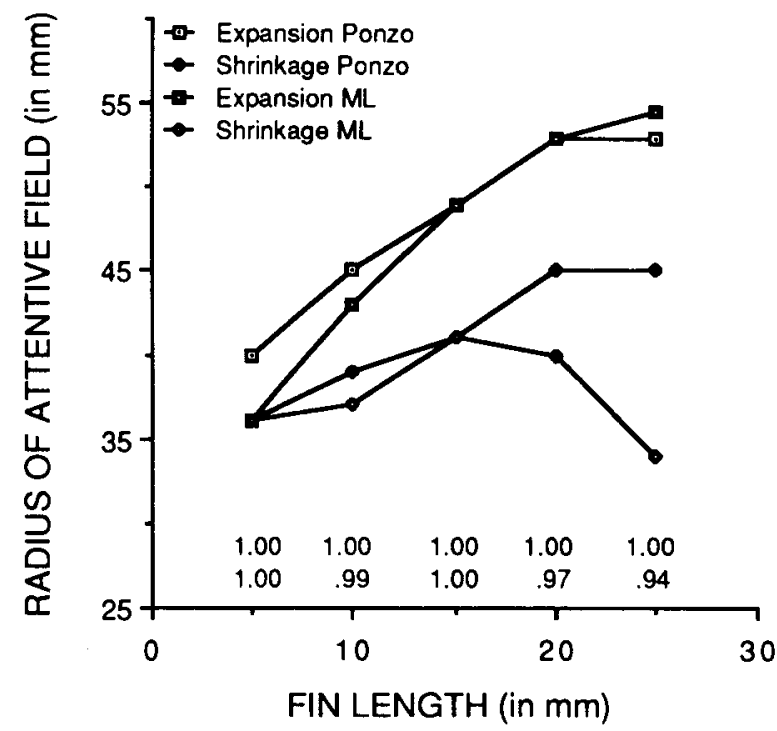

Figure 13. Effects of fin length on radii of inferred attentive fields surrounding shrinkage and expansion fins in Ponzo and Müller-Lyer configurations. The numbers above the abscissa are correlation coefficients that represent the best fit between theoretical and real data. The coefficients for the Ponzo pattern are in the upper row, and for the Müller-Lyer figure in the lower row.

the sum of its parts is that wholes are characterized by emergent properties that do not exist in any of the parts and are therefore not predictable from a complete knowledge of the parts alone.

Consider the proposition that the effect of increasing the length of a shrinkage fin will be different when that fin is coupled with another shrinkage fin (Müller-Lyer pattern) than when it is coupled with an expansion fin (Ponzo pattern). At first blush, it sounds as if we are dealing with an emergent property because (1) that property cannot be predicted from a complete knowledge of how shrinkage fins alone operate and (2) it cannot be predicted from a complete knowledge of how expansion fins alone operate. However, it might be argued that although, in a narrow sense, it is self-evident that no amount of empirical data on expansion fins alone can tell us directly what will occur in the shrinkage fins, it might be possible to do so indirectly. Thus, a complete knowledge of the operation of expansion fins will include the knowledge that they drive a third factor (circular attentive field size) in such a way as to increase that factor as fin length increases. From this knowledge it would follow that if a shrinkage fin were coupled with an expansion fin, the effect of the shrinkage fin would be modified by attentive fields that are driven by that expansion fin. In this sense, then, an emergent property is predictable from a knowledge of one of its parts, but that knowledge is of a special kind. It is knowledge of how one part interacts with a system as a whole that allows us to predict how a second part will interact with the first. In other words, it may not be possible to predict the whole from a complete knowledge of the parts, but a complete knowledge of the parts might be attainable from a knowledge of the whole.

\section{REFERENCES}

Brigell, M., Uhlarik, J., \& Goldhorn, P. (1977). Contextual influences and illusions of extent. Journal of Experimental Psychology: Human Perception \& Performance, 3, 105-118.

GREGORY, R. L. (1963). Distortion of visual space as inappropriate constancy scaling. Nature, 199, 678-680.

JAEGER, T. (1985). Contextual effects in the parallel lines illusion: Some implications for assimilation theory. Perceptual \& Motor Skills, 61, 1263-1273.

JoRDAN, K., ENGLISH, P. W. (1989). Simultaneous sampling and length contrast. Perception \& Psychophysics, 46, 546-554.

Jordan, K., RANDALL, J. (1987). The effects of framing ratio and oblique length on Ponzo illusion magnitude. Perception \& Psychophysics, 41, 435-439.

KüNNAPAS, T. M. (1955). Influence of frame size on apparent length of a line. Journal of Experimental Psychology, 50, 168-170.

LARSEN, J. D., GARN, N. K. (1988). Wings in the intershaft space contribute to the Mueller-Lyer illusion. Perceptual \& Motor Skills, 67, 831-834.

Mackworth, N. H. (1965). Visual noise causes tunnel vision. Psychonomic Science, 3, 67-68.

McClellan, P. G., Bernstein, I. H., Garbin, C. P. (1984). What makes the Mueller a liar: A multiple-cue approach. Perception \& Psychophysics, 36, 234-244.

Ponzo, M. (1912). Rapports entre quelques illusions visuelles de contraste angulaire et l'appréciation de grandeur des astres à l'horizon. Archives laliennes de Biologie, 58, 327-329.

Pressey, A. W. (1971). An extension of assimilation theory to illusions of size, area, and direction. Perception \& Psychophysics, 9, 172-176.

Pressey, A. W. (1972). The assimilation theory of geometric illusions: An additional postulate. Perception \& Psychophysics, 11, 28-30.

Pressey, A. W. (1974a). Effect of size of angle on the ambiguous Müller-Lyer illusion. Acta Psychologica, 38, 401-404.

Pressey, A. W. (1974b). Evidence for the role of attentive fields in the perception of illusions. Quarterly Journal of Experimental Psychology, 26, 464-471.

Pressey, A. W., Butchard, N., \& SCrivner, L. (1971). Assimilation theory and the Ponzo illusion: Quantitative predictions. Canadian Journal of Psychology, 25, 486-497.

Pressey, A. W., \& Di Lollo, V. (1978). Effects of distance between standard and comparison lines on the Müller-Lyer illusion. Perception \& Psychophysics, 24, 415-419.

Pressey, A. W., Di Lollo, V., \& Tait, R. W. (1977). Effects of gap size between shaft and fins and of angle of fins on the Müller-Lyer illusion. Perception, 6, 435-439.

Pressey, A. W., Kersten, B. (1989). Attention and the Müller-Lyer illusion: Simulation of an experiment by Larsen and Garn. Perceptual \& Motor Skills, 68, 1323-1329.

Pressey, A. W., Murray, R. (1976). Further developments in the assimilation theory of geometric illusions: The adjacency principle. Perception \& Psychophysics, 19, 536-544.

Pressey, A. W., Pressey, C. A. (1992). Attentional fields are related to focal and contextual features: A study of Müller-Lyer distortions. Perception \& Psychophysics, 51, 423-436.

Pressey, A. W., \& SMith MArTin, N. (1990). The effects of varying fins in Müller-Lyer and Holding illusions. Psychological Research, 52, 46-53.

Pressey, A. W., \& Wilson, A. E. (1980). Assimilation theory and the Baldwin illusion. Italian Journal of Psychology, 7, 65-73.

Reardon, M. E., Parks, T. E. (1983). The Ponzo illusion without suggested depth. American Journal of Psychology, 96, 107-112.

Restle, F., \& Decker, J. (1977). Size of the Mueller-Lyer illusion as a function of its dimensions: Theory and data. Perception \& Psychophysics, 21, 489-503.

SANDERS, A. (1963). The selective process in the functional visual field. Soesterberg, The Netherlands: Institute for Perception, RVO-TNO.

Sekuler, R., Blake, R. (1990). Perception. New York: McGraw-Hill.

TAusCh, R. (1954). Optische Täuschungen als artifizielle Effekte der Gestaitungsprozesse von Grössen- und Formenkonstanz in der 
natürlichen Raumwahrnehmung. Psychologische Forschung, 24, 299-348.

Waite, H., \& Massaro, D. W. (1970). Test of Gregory's constancy scaling explanation of the Müller-Lyer illusion. Nature, 227, 733-734.

WARREN, R. M., BAShFoRD, J. A. (1977). Müller-Lyer illusions: Their origin in processes facilitating object recognition. Perception, 6, 615-626.

Weintraub, D. J., \& Krantz, D. H. (1971). The Poggendorff illusion: Amputations, rotations, and other perturbations. Perception \& Psychophysics, 10, 257-264.

\section{NOTES}

1. The phrase "attentive field" was selected early in the development of integrative field theory to differentiate it from ideas proposed by Mackworth (1965) and Sanders (1963). It is defined as a spatial area of a display that represents differing probabilities of being sampled while the task is being performed. Although eye movements are undoubtedly involved in such sampling of information, the construct is not reducible to such eye movements because attention can be spatially distributed while the eyes are fixed. Thus, eye movements are correlated with, but are not identical to, the attentive field.

2. Stanley Coren brought this effect to my attention in November 1982. The effect is a real-object version of Wundt's area illusion, an analysis of which was provided from the perspective of integrative field theory in 1971 (Pressey, 1971).

3. Equation 6 differs from earlier equations because it deals with lengths of lines rather than with differences between contextual and focal lengths. However, an illusion score can be obtained as before by subtracting the length of the focal line from $J_{s}$ in Equation 6 .

(Manuscript received July 16, 1991; revision accepted for publication February 18, 1992.) 